\title{
Técnica e compreensão do uso dos dispositivos inalatórios em pacientes com asma ou DPOC***
}

\author{
Knowledge of and technique for using inhalation \\ devices among asthma patients and COPD patients
}

\author{
Maria Luiza de Moraes Souza, Andrea Cristina Meneghini, Érica Ferraz, \\ Elcio Oliveira Vianna, Marcos Carvalho Borges
}

\begin{abstract}
Resumo
Objetivo: Avaliar a compreensão e a técnica de uso dos dispositivos inalatórios prescritos de pacientes com asma ou DPOC atendidos em um hospital-escola terciário. Métodos: Os pacientes foram avaliados na sala de pós-consulta sem que o médico soubesse do estudo. Foi solicitado que o paciente demonstrasse como utiliza a medicação inalatória e, em seguida, esse foi entrevistado sobre a compreensão dos dispositivos inalatórios, controle da doença e orientações recebidas durante as consultas. Resultados: Foram avaliados 120 voluntários: 60 asmáticos e 60 com DPOC. Todos os asmáticos e 98,3\% do grupo DPOC relataram saber utilizar os medicamentos inalatórios. $\mathrm{Na}$ amostra como um todo, 113 pacientes $(94,2 \%)$ cometeram pelo menos um erro ao utilizar o dispositivo inalatório. Os pacientes cometeram mais erros ao utilizar aerossol dosimetrado do que ao utilizar os inaladores de pó seco Aerolizer $^{\circledR}(p<0,001)$ ou Pulvinal ${ }^{\circledR}(p<0,001)$, assim como mais erros ao utilizar Aerolizer ${ }^{\circledR}$ do que ao utilizar Pulvinal ${ }^{\circledR}(p<0,05)$. 0 grupo DPOC cometeu significativamente mais erros que o grupo asma ao utilizar o aerossol dosimetrado ( $p=0,0023)$, Pulvinal ${ }^{\circledR}(p=0,0065)$ e Aerolizer ${ }^{\circledR}(p=0,012)$. Conclusões: Embora a maioria dos pacientes relatasse saber a técnica adequada de utilização dos dispositivos inalatórios, 94,2\% cometeu pelo menos um erro na utilização dos dispositivos, demonstrando técnica insatisfatória e discrepância entre a compreensão e a prática. Portanto, apenas questionar os pacientes sobre o uso dos dispositivos inalatórios não é suficiente. Medidas práticas devem ser tomadas a fim de diminuir os erros e otimizar a terapêutica.
\end{abstract}

Descritores: Inaladores dosimetrados; Nebulizadores e vaporizadores; Asma;

Doença pulmonar obstrutiva crônica.

\begin{abstract}
Objective: To evaluate knowledge of and techniques for using prescribed inhalation devices among patients with asthma or COPD treated at a tertiary teaching hospital. Methods: Patients were assessed after medical visits, and their physicians were blinded to this fact. Patients were asked to demonstrate their inhaler technique and were then interviewed regarding their knowledge of inhalation devices, control of the disease and instructions received during medical visits. Results: We included 120 volunteers: 60 with asthma and 60 with COPD. All of the asthma patients and 98.3\% of the COPD patients claimed to know how to use inhaled medications. In the sample as a whole, 113 patients $(94.2 \%)$ committed at least one error when using the inhalation device. Patients committed more errors when using metered-dose inhalers than when using the dry-powder inhalers Aerolizer ${ }^{\circledR}(p<0.001)$ or Pulvinal ${ }^{\circledR}(p<0.001)$, as well as committing more errors when using the Aerolizer ${ }^{\circledR}$ inhaler than when using the Pulvinal ${ }^{\circledR}$ inhaler $(p<0.05)$. Using the metered-dose, Pulvinal ${ }^{\circledR}$ and Aerolizer ${ }^{\circledR}$ inhalers, the COPD group patients committed more errors than did the asthma group patients $(p=0.0023, p=0.0065$ and $p=0.012$, respectively). Conclusions: Although the majority of the patients claimed to know how to use inhalation devices, the fact that 94.2\% committed at least one error shows that their technique was inappropriate and reveals a discrepancy between understanding and practice. Therefore, it is not sufficient to ask patients whether they know how to use inhalation devices. Practical measures should be taken in order to minimize errors and optimize treatment.
\end{abstract}

Keywords: Metered dose inhalers; Nebulizers and vaporizers; Asthma; Pulmonary disease, chronic obstructive.

\footnotetext{
* Trabalho realizado no Departamento de Clínica Médica, Faculdade de Medicina de Ribeirão Preto, Universidade de São Paulo, Ribeirão Preto (SP) Brasil.

Endereço para correspondência: Elcio O. Vianna. Divisão de Pneumologia, Departamento de Clínica Médica, Faculdade de Medicina de Ribeirão Preto, Universidade de São Paulo, Av. Bandeirantes, 3900, CEP 14048-900, Ribeirão Preto, SP, Brasil.

Tel 5516 3602-2706. Fax 5516 3633-6695. E-mail: evianna@uol.com.br

Apoio financeiro: Nenhum.

Recebido para publicação em 19/3/2009. Aprovado, após revisão, em 19/5/2009.

** Os anexos estão disponíveis integralmente junto à versão eletrônica deste artigo em www.jornaldepneumologia.com.br
} 


\section{Introdução}

A asma e a DPOC são doenças respiratórias que apresentam elevadas prevalências e estão presentes em todos os países, independentemente de seu grau de desenvolvimento, sendo reconhecidas como um problema de saúde pública mundial.

No Brasil, a prevalência de asma em crianças oscila entre $18 \%$ e $27 \% .^{(1,2)}$ Ocorrem anualmente 350 mil internações por asma no Brasil, constituindo a quarta causa de hospitalização no Sistema Único de Saúde (SUS) - 2,3\% do total e a terceira causa de hospitalização entre crianças e adultos jovens. ${ }^{(3)}$ Em 2004, foram internadas 367 mil pessoas, e ocorreram mais de 2.000 óbitos devido à asma, com gastos de aproximadamente 123,2 milhões de reais para o SUS.(4) Com o tratamento, busca-se obter o melhor controle possível da asma, sendo utilizados inicialmente medicamentos por via inalatória, como corticosteroides e broncodilatadores.

Não se conhece a real prevalência da DPOC em nosso meio. Estima-se que seja em torno de $12 \%$ em adultos. ${ }^{(5)}$ Em um estudo realizado na cidade de São Paulo, a prevalência da DPOC variou entre $6,0 \%$ e $15,8 \%$ em indivíduos acima de 40 anos. $^{(6)}$ A DPOC é uma das mais importantes causas de mortalidade na maioria dos países, tendo ocupado a sexta posição em 1990, e previsões apontam que se tornará a terceira causa de mortalidade no mundo em 2020. ${ }^{(7)}$ No Brasil, a DPOC ocupa da quarta à sétima posição entre as principais causas de mortalidade, sendo que o número de óbitos consequentes a ela vem aumentando nos últimos 20 anos em ambos os sexos. ${ }^{(5)}$ Em 2003, foi a quinta principal causa de internação de indivíduos acima de 40 anos, com um gasto de 72 milhões de reais. ${ }^{(5)}$ Assim como na asma, o tratamento visa obter o melhor controle possível da DPOC, sendo também utilizados inicialmente medicamentos por via inalatória, como broncodilatadores e corticosteroides.

Os medicamentos inalatórios são administrados diretamente nas vias aéreas e propiciam maior concentração local e menor risco de efeitos colaterais sistêmicos. ${ }^{\left({ }^{(8)}\right.}$ Durante anos, os nebulizadores de jato foram os únicos dispositivos inalatórios disponíveis; porém, o surgimento de outros dispositivos (aerossóis dosimetrados, com e sem espaçadores, e inaladores de pó) permitiu melhorar a oferta de drogas para o pulmão e diminuir os efeitos colaterais locais e sistê- micos. ${ }^{(9,10)}$ Preferências individuais, conveniência, facilidade de uso e fatores econômicos podem influenciar a eficiência, a adesão ao tratamento e o controle da doença. Programas de educação, tanto para crianças quanto para adultos, têm papel central no treinamento de pacientes para a aquisição da técnica correta de utilização dos dispositivos inalatórios. ${ }^{(11-13)} \mathrm{Um}$ programa brasileiro de educação em asma, com um modelo de pós-consulta padronizado, evidenciou melhora na identificação de problemas relacionados ao manejo da asma e nos índices de qualidade de vida. ${ }^{(14)}$

A terapia inalatória tem papel central no tratamento de pacientes com asma e DPOC, e a sua utilização requer um treinamento supervisionado e contínuo. ${ }^{(8,15,16)} 0$ objetivo deste estudo foi avaliar o conhecimento prático e a compreensão de voluntários com asma e DPOC, atendidos em um hospital-escola terciário, em relação ao uso dos dispositivos inalatórios.

\section{Métodos}

Estudo observacional realizado entre os meses de setembro de 2008 e janeiro de 2009. A amostra constituiu de 120 pacientes, 60 com asma (grupo asma) e 60 com DPOC (grupo DPOC), atendidos nos ambulatórios do Hospital das Clínicas da Faculdade de Medicina de Ribeirão Preto da Universidade de São Paulo (HCFMRP-USP), que faziam seguimento crônico, com retorno, em geral, trimestral. Os pacientes apresentavam asma ou DPOC, definidas conforme as IV Diretrizes Brasileiras para o Manejo da Asma $^{(15)}$ e o 11 Consenso Brasileiro sobre Doença Pulmonar Obstrutiva Crônica, ${ }^{(5)}$ respectivamente, e faziam uso contínuo de medicação inalatória. Os voluntários foram incluídos no estudo após leitura e assinatura do termo de consentimento livre e esclarecido aprovado previamente pelo Comitê de Ética em Pesquisa do HCFMRP-USP.

Todos os pacientes foram avaliados na sala de pós-consulta da enfermagem, por uma aluna de medicina do sexto ano e uma fisioterapeuta, ambas previamente treinadas, logo após a consulta médica de rotina, sem que o médico soubesse do estudo. Inicialmente, foi realizada uma avaliação prática do uso do dispositivo inalatório, solicitando que o paciente demonstrasse o uso da medicação inalatória. 0 observador preenchia uma ficha validada pela literatura para a conferência do uso desse 
dispositivo (Anexo 1 na versão online). ${ }^{(10,17-19)}$ Estavam disponíveis os seguintes dispositivos contendo placebo: aerossol dosimetrado, Pulvinal ${ }^{\circledR}$, Aerolizer $^{\circledR}$, Handhaler $^{\circledR}$, Turbuhaler $^{\circledR}$ e Diskus ${ }^{\circledR}$. Posteriormente, os participantes foram entrevistados sobre sua compreensão do uso dos dispositivos inalatórios, controle da doença e orientações recebidas durante as consultas de rotina (Anexo 2 na versão online). Após as avaliações, foi feita uma instrução verbal sobre a utilização correta dos dispositivos.

A comparação da quantidade de erros cometidos pelos pacientes ao utilizar os diferentes dispositivos foi feita pelo teste de Kruskal-Wallis. A comparação da quantidade de erros cometidos pelo grupo asma e pelo grupo DPOC foi feita separadamente para cada dispositivo através do teste de Mann-Whitney. Em relação ao questionário, as respostas obtidas em cada questão do grupo asma foram comparadas com as do grupo DPOC pelo teste exato de Fisher. Em todas as análises, foi considerada como significância estatística um valor de $p \leq 0,05$.

\section{Resultados}

Foram avaliados 60 asmáticos (grupo asma), com idades entre 18 e 72 anos (média = $48 \pm 12$ anos); 17 (28,3\%) eram do sexo mascu- lino e $43(71,7 \%)$ do sexo feminino. Desses, 54 asmáticos $(90,0 \%)$ utilizavam corticosteroide inalatório, $41(68,3 \%) \quad \beta_{2}$-agonista de longa duração e $44(73,3 \%) \beta_{2}$-agonista de curta duração. 0 grupo DPOC foi constituído por 60 pacientes com idades entre 47 e 91 anos (média $=65 \pm 10$ anos); 39 (65,0\%) eram do sexo masculino e $21(35,0 \%)$ do sexo feminino. Desses, 29 pacientes $(48,3 \%)$ utilizavam corticosteroide inalatório, $53(88,3 \%) \quad \beta_{2}$-agonista de longa duração, $25(41,7 \%) \beta_{2}$-agonista de curta duração e 2 (3,33\%) anticolinérgico de longa duração. Todos os pacientes responderam o questionário. A Tabela 1 mostra as porcentagens de respostas positivas encontradas em cada questão sobre o uso dos medicamentos inalatórios no grupo asma e no grupo DPOC.

Comparando as respostas obtidas nos dois grupos, cinco questões apresentaram diferenças significativas entre suas respostas. 0 grupo asma, em comparação com o grupo DPOC, relatou que, com mais frequência, o médico (ou outro profissional de saúde) já havia ensinado o modo correto do uso da medicação $(p=0,05)$, havia visto o paciente usar corretamente a medicação ( $p<0,0001)$, reavaliava em toda consulta $o$ uso da medicação $(p=0,01)$ e já havia visto o paciente utilizando a medicação cinco vezes ou

Tabela 1 - Porcentagem de respostas positivas às questões do questionário sobre os medicamentos inalatórios de acordo com o grupo de pacientes.

\begin{tabular}{|c|c|c|c|}
\hline \multirow[t]{2}{*}{ Questões } & \multicolumn{2}{|c|}{$\begin{array}{l}\text { \% de respostas } \\
\text { positivas }^{\mathrm{a}}\end{array}$} & \multirow[t]{2}{*}{$\mathrm{p}$} \\
\hline & Asma & DPOC & \\
\hline 1. Você sabe utilizar as medicações inalatórias que foram prescritas? & 100,0 & 98,3 & 1,00 \\
\hline 2. Você tem dúvidas quanto ao modo de utilizar as medicações inalatórias? & 5,0 & 8,3 & 0,72 \\
\hline 3. Você acha que as medicações inalatórias têm bom resultado? & 86,7 & 94,9 & 0,66 \\
\hline 4. Você acha que é importante a técnica ou o modo como você usa a medicação? & 96,6 & 96,7 & 1,00 \\
\hline $\begin{array}{l}\text { 5. Seu médico (ou profissional da saúde) já ensinou o modo correto de usar as } \\
\text { medicações inalatórias? }\end{array}$ & 90,0 & 75,0 & 0,05 \\
\hline 6. Seu médico já viu você usando sua medicação inalatória? & 66,7 & 26,7 & $<0,0001$ \\
\hline 7. Seu médico reavalia em toda consulta como você usa a medicação inalatória? & 40,7 & 17,3 & 0,01 \\
\hline \multicolumn{4}{|l|}{ 8. Quantas vezes seu médico viu como você usa a medicação inalatória? } \\
\hline Nenhuma vez & 30,5 & 69,2 & $<0,0001$ \\
\hline $1 \mathrm{vez}$ & 20,3 & 19,2 & 1,00 \\
\hline 2 vezes & 11,9 & 3,8 & 0,17 \\
\hline 3 vezes & 13,6 & 3,8 & 0,10 \\
\hline 4 vezes & 5,1 & 1,9 & 0,62 \\
\hline 5 ou mais vezes & 18,6 & 1,9 & 0,005 \\
\hline 9. Você acha que sua doença está bem controlada? & 80,0 & 78,0 & 0,83 \\
\hline
\end{tabular}


Tabela 2 - Número absoluto e porcentual de pacientes com asma e DPOC que cometeu pelo menos um erro ao utilizar os dispositivos inalatórios.

\begin{tabular}{lcc}
$\begin{array}{c}\text { Tipo de } \\
\text { dispositivo }\end{array}$ & $\begin{array}{c}\text { Grupo asma } \\
(\mathrm{n}=60)\end{array}$ & $\begin{array}{c}\text { Grupo DPOC } \\
(\mathrm{n}=60)\end{array}$ \\
\hline $\begin{array}{l}\text { Aerossol } \\
\text { dosimetrado }\end{array}$ & $42(95,5 \%)$ & $22(95,7 \%)$ \\
Aerolizer $^{\circledR}$ & $41(87,2 \%)$ & $49(90,7 \%)$ \\
Pulvinal $^{\circledR}$ & $25(61,0 \%)$ & $13(92,9 \%)$ \\
Handhaler $^{\circledR}$ & - & $2(100,0 \%)$ \\
Turbuhaler $^{\circledR}$ & $1(100,0 \%)$ & $1(100,0 \%)$ \\
Diskus $^{\circledR}$ & - & - \\
\hline
\end{tabular}

mais $(p=0,005)$. Além disso, aproximadamente 70\% dos pacientes do grupo DPOC, em comparação com 30\% dos do grupo asma ( $p<0,0001)$ responderam que o médico nunca os havia visto utilizando a medicação inalatória.

Em relação aos asmáticos, $12(20,0 \%)$ utilizavam apenas um dispositivo; 23 (38,3\%), dois dispositivos; e 25 (41,7\%), três dispositivos, distribuídos na seguinte proporção: 47 (78,3\%) utilizavam Aerolizer ${ }^{\circledR} ; 44$ (73,3\%), aerossol dosimetrado; 41 (68,3\%), Pulvinal ${ }^{\circledR}$; e 1 (1,7\%),

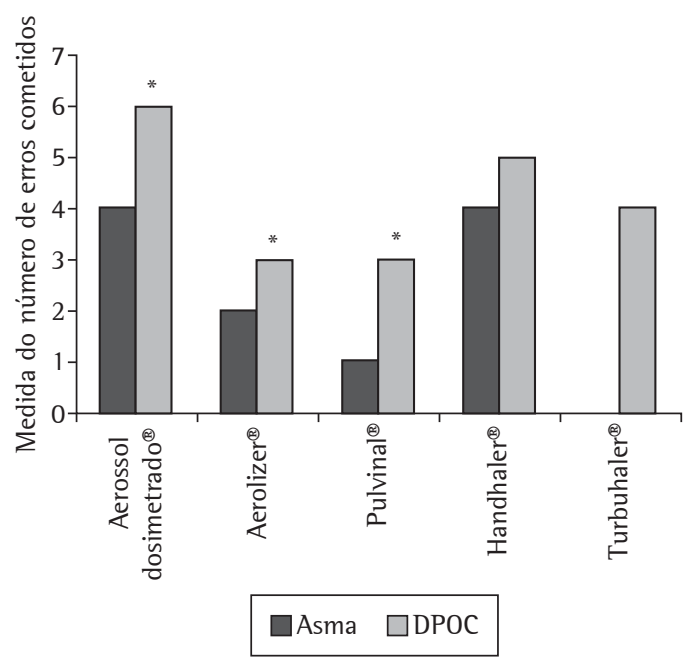

Figura 1 - Média do número de erros cometidos pelos 60 pacientes com asma e pelos 60 pacientes com DPOC ao utilizar os dispositivos inalatórios. Os pacientes com DPOC cometeram mais erros que os asmáticos ao utilizarem o aerossol dosimetrado $(p=0,0021)$, Aerolizer $^{\circledR}(p=0,011)$ e Pulvinal ${ }^{\circledR}(p=0,0053)$. A comparação estatística dos dispositivos Handhaler ${ }^{\circledR} \mathrm{e}$ Turbuhaler $^{\circledR}$ não foi possível pois apenas 2 pacientes faziam uso desses dispositivos.
Turbuhaler ${ }^{\circledR}$. Apenas $5(11,4 \%)$ usavam espaçador no aerossol dosimetrado. Dos pacientes com DPOC, 33 (55,0\%) utilizavam apenas um dispositivo; 20 (33,3\%), dois dispositivos; e 7 (11,7\%); três dispositivos. Desses, 54 (90,0\%) utilizavam Aerolizer $^{\circledR} ; 23$ (38,3\%), aerossol dosimetrado; 14 (23,3\%), Pulvinal ${ }^{\circledR} ; 2$ (3,3\%), Handhaler ${ }^{\circledR}$; e $1(1,7 \%)$, Turbuhaler ${ }^{\circledR}$. Apenas $5(21,7 \%)$ usavam espaçador no aerossol dosimetrado.

Do total da amostra, 113 pacientes (94,2\%) cometeram, pelo menos, um erro ao utilizar o dispositivo inalatório. Portanto, somente 2 pacientes com asma e 5 com DPOC realizaram corretamente todos os passos ao utilizar a medicação inalatória. 0 grupo DPOC cometeu mais erros que o grupo asma $(p<0,0001)$ ao utilizar os dispositivos inalatórios. A porcentagem de erros cometidos pelo grupo asma e pelo grupo DPOC no uso dos diferentes dispositivos encontra-se na Tabela 2 e na Figura 1.

Os pacientes cometeram maior frequência de erros ao utilizar o aerossol dosimetrado quando comparado ao Aerolizer ${ }^{\circledR}(p<0,001)$ e Pulvinal ${ }^{\circledR}$ ( $p<0,001)$, e mais erros ao utilizar o Aerolizer $^{\circledR}$ quando comparado ao Pulvinal ${ }^{\circledR}(p<0,05$; Figura 2). Não houve diferença na quantidade de erros cometidos pelos pacientes quando foram comparados o Handhaler ${ }^{\circledR}$ e o Turbuhaler $^{\circledR}$; porém, apenas 2 pacientes faziam uso desses dispositivos. A comparação entre o grupo DPOC e o grupo asma, em relação aos diferentes dispositivos, evidenciou que os pacientes com DPOC cometeram significativamente mais erros que os asmáticos ao utilizar o aerossol dosimetrado $(p=0,0021)$, Pulvinal $^{\circledR}(p=0,0053)$ e Aerolizer ${ }^{\circledR}$ $(p=0,011)$.

Dentre os erros mais cometidos por todos os pacientes nos diferentes dispositivos, foi observado que, ao utilizar o Aerolizer ${ }^{\circledR}, 55$ pacientes $(54,5 \%)$ não realizavam uma expiração adequada antes da inalação do medicamento. No caso do aerossol dosimetrado, 49 pacientes $(73,1 \%)$ não mantinham o inalador à distância correta dos lábios, $41(61,2 \%)$ não realizavam expiração adequada antes da inalação do medicamento, $28(41,8 \%)$ não agitavam o inalador antes da utilização, e 23 (34,3\%) não inalavam lentamente durante o procedimento. No caso do Pulvinal ${ }^{\circledR}, 27$ pacientes $(49,1 \%)$ não realizavam uma expiração adequada, e $9(16,4 \%)$ não rodavam o inalador até ouvir o clique. 


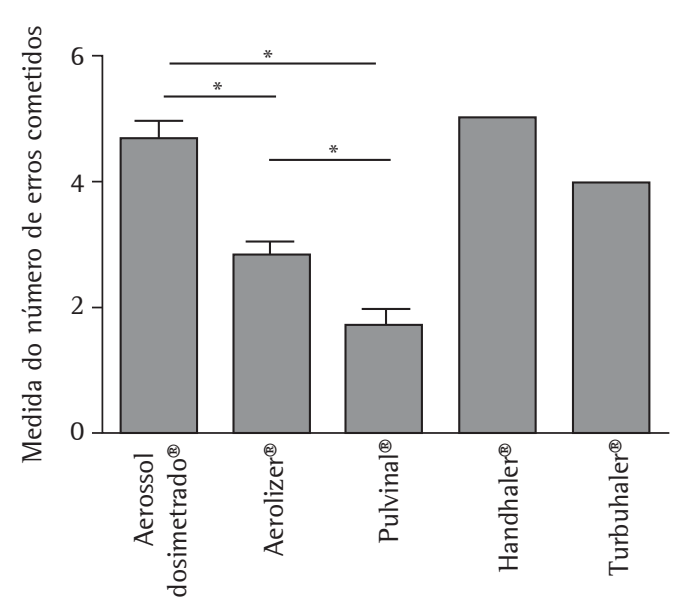

Figura 2 - Média do número de erros cometidos pelos 120 pacientes com asma ou com DPOC ao utilizar os diferentes dispositivos inalatórios. A comparação estatística dos dispositivos Handhaler ${ }^{\circledR}$ e Turbuhaler ${ }^{\circledR}$ não foi possível pois apenas 2 pacientes faziam uso destes dispositivos. ${ }^{*} p<0,05$.

\section{Discussão}

Todos os pacientes com asma e quase todos (98\%) com DPOC relataram conhecer a técnica adequada de utilização dos dispositivos inalatórios. Apesar disso, a maioria dos pacientes (94,2\%), tanto os asmáticos como os com DPOC, cometeu pelo menos um erro na utilização dos dispositivos, demonstrando o uso de técnica insatisfatória e a discrepância entre a compreensão do paciente e seu conhecimento prático real.

A utilização errônea dos dispositivos pode resultar em uma terapêutica inadequada das doenças respiratórias. ${ }^{(20,21)}$ Existem inúmeros dispositivos disponíveis para a terapia inalatória, o que possibilita a utilização dos medicamentos em diversos modos. É inegável o benefício de adequar o dispositivo ao paciente; porém, a prescrição médica e o conhecimento prático do paciente podem ser prejudicados se não forem adequadamente revistos.

Alguns estudos avaliaram o uso dos medicamentos inalatórios em diversas populações, com resultados diferentes. Alguns autores analisaram a técnica de uso de diferentes dispositivos em pacientes na "vida real" e constataram que os dois erros mais comuns foram não realizar uma expiração adequada $(28,9 \%)$ e não realizar uma pausa inspiratória $(28,3 \%)$. Dos pacientes avaliados, 66\% tiveram pelo menos um erro com o dispositivo pressurizado, e 49-55\% tiveram pelo menos um erro com os dispositivos inalatórios "ativados pela inalação" (pó). ${ }^{(17)}$ Em um estudo espanhol, a avaliação da técnica inalatória entre mais de 1.640 voluntários europeus (746 pacientes, 466 enfermeiras e 428 médicos) evidenciou a utilização adequada do aerossol dosimetrado em apenas 9\% dos pacientes, 15\% das enfermeiras e $28 \%$ dos médicos. ${ }^{(18)}$ Outros autores, ao avaliarem 4.078 asmáticos, detectaram que $71 \%$ tinham dificuldades para utilizar aerossóis dosimetrados e que o uso incorreto estava associado à asma menos controlada. ${ }^{(21)}$

No Brasil, avaliou-se a técnica de uso dos aerossóis dosimetrados e dos inaladores de pó de 40 asmáticos, 20 estudantes de medicina do sexto ano e 36 médicos residentes. Os autores do estudo observaram que entre 33\% e 40\% dos voluntários de cada grupo cometeram erros ao utilizar os aerossóis dosimetrados, o mesmo ocorrendo com 4-12\% dos mesmos voluntários ao utilizar os inaladores de pó. Porém, não houve diferença significante entre os grupos ou dispositivos. ${ }^{(22)}$

A falha terapêutica em pacientes asmáticos ou com DPOC pode ocorrer devido à utilização incorreta dos medicamentos inalatórios decorrente de diversos fatores, como falta de orientação, orientação inadequada e condições de aprendizado dos pacientes. ${ }^{(7,8,23)}$ Alguns autores demonstraram que o conhecimento a respeito do uso de aerossóis dosimetrados entre os profissionais de saúde em um hospital brasileiro de referência em saúde da criança era insatisfatório. ${ }^{(10)}$ Outros autores detectaram que apenas 30\% dos médicos residentes demonstravam corretamente a técnica de uso de um dispositivo inalatório para pacientes com asma não-controlada. ${ }^{(24)}$ Além disso, a técnica para a utilização de medicação inalatória deve ser constantemente reavaliada, pois alguns pacientes podem não realizar uma técnica adequada mesmo após diversas orientações, e a técnica correta pode se tornar inadequada com o passar do tempo. ${ }^{(23,25)}$ Alguns autores avaliaram prospectivamente 93 pacientes com DPOC ou asma que nunca haviam feito uso de medicação inalatória por inalador dosimetrado e, após 10 dias da primeira explicação, somente $48,4 \%$ dos pacientes realizaram a técnica de uso do dispositivo corretamente. ${ }^{(26)}$ 
Os resultados expostos demonstram uma técnica de uso dos dispositivos inalatórios insatisfatória, tanto em pacientes com asma quanto naqueles com DPOC em um hospital-escola terciário. 0 fato dos pacientes tenderem a sempre responder que sabem utilizar os dispositivos pode ser responsável por um círculo vicioso, no qual o paciente afirma sabê-lo, e a equipe, por acreditar nele, não o testa. Essa explicação é reforçada pelo baixo número de médicos que avalia e, sobretudo, reavalia a técnica de uso dos dispositivos. Cabe ressaltar que, em nosso serviço, nenhum outro profissional de saúde, além do médico, avalia a técnica de uso da medicação inalatória, e ainda não existe um programa estruturado de educação para os pacientes. Outros fatores podem ter contribuído para o uso de técnica inadequada, como o nível socioeconômico dos pacientes e a orientação ser realizada por médicos residentes em treinamento. ${ }^{(24)}$

Os pacientes com DPOC cometeram mais erros que os asmáticos. Uma possível explicação para esse fato pode ser que muitos dos asmáticos foram avaliados em um ambulatório especializado em asma, onde somente residentes de pneumologia fazem o atendimento. Já os pacientes com DPOC foram atendidos tanto por residentes de pneumologia quanto por residentes de clínica médica geral. Essa teoria é reforçada pelas respostas obtidas no questionário, que demonstraram que os asmáticos foram mais bem orientados quanto ao uso dos medicamentos inalatórios. Outras explicações podem ser devido a diferenças nas características dos dois grupos, como idade, condição socioeconômica e tempo da doença. Outros estudos são necessários para um melhor esclarecimento. Apesar dos pacientes com DPOC terem cometido mais erros que os asmáticos, a técnica de utilização dos dispositivos inalatórios demonstrada pelos asmáticos foi também insatisfatória.

Dos dispositivos testados, foi encontrado um maior número de erros no uso de aerossol dosimetrado, Aerolizer ${ }^{\circledR}$ e Pulvinal ${ }^{\circledR}$, nessa sequência. Devido ao pequeno número de pacientes que faziam uso de Turbuhaler $^{\circledR}$ e Handhaler ${ }^{\circledR}$, os resultados referentes a esses dispositivos devem ser analisados com cautela. Apesar da diferença no número de erros no uso dos dispositivos, não é possível, neste estudo, relacionar esse achado com o controle clínico dos pacientes. Se possível, deve-se evitar a utilização de dispositivos diferentes para um mesmo paciente. ${ }^{(27)}$

Um dado interessante é o pequeno número de pacientes com asma ou com DPOC que utilizavam o espaçador. Isso pode decorrer de um conceito errôneo de que a função do espaçador é apenas facilitar o uso de medicações inalatórias. Outra função do espaçador é permitir que partículas grandes que não atingem as vias aéreas distais fiquem retidas no aparelho e não na orofaringe, e que partículas menores sejam inaladas, atingindo as vias aéreas distais, possibilitando melhores resultados terapêuticos e menor risco de absorção sistêmica. ${ }^{(10,28)}$ Recentemente, alguns estudos têm evidenciado equivalência clínica dos aerossóis dosimetrados com hidrofluoralcano (HFA) sem espaçador quando comparados com aerossóis dosimetrados com clorofluorcarbono (CFC) e com espaçador. ${ }^{(29)}$ Em nosso estudo, não foi avaliado se os aerossóis dosimetrados eram com CFC ou HFA.

Como o controle das doenças respiratórias depende também da utilização correta das medicações inalatórias, é fundamental que medidas práticas sejam realizadas a fim de miminizar os erros e aumentar a eficácia das medicações. Inúmeras medidas podem ser adotadas, como a reavaliação prática da técnica pelo médico em diversas consultas, a despeito de o paciente afirmar que sabe realizá-la, assim como a reavaliação prática pela equipe de saúde na pós-consulta e a implementação de programas de educação de curto ou longo prazo. ${ }^{(30)}$ É indiscutível a necessidade do descobrimento de novas medicações para o tratamento das doenças respiratórias, mas é imprescindível que as medicações inalatórias disponíveis sejam adequadamente utilizadas antes de se definir que uma doença não está controlada ou está parcialmente controlada e se iniciar medidas terapêuticas novas e caras. ${ }^{(23)}$

Os dados evidenciam uma discrepância entre a compreensão do paciente e a prática do uso dos dispositivos inalatórios, cuja técnica é insatisfatória, tanto em pacientes com asma quanto em pacientes com DPOC. Medidas práticas devem ser tomadas a fim de diminuir os erros cometidos pelos pacientes ao utilizar as medicações inalatórias, proporcionando um melhor controle das doenças respiratórias. 


\section{Referências}

1. Worldwide variation in prevalence of symptoms of asthma, allergic rhinoconjunctivitis, and atopic eczema: ISAAC. The International Study of Asthma and Allergies in Childhood (ISAAC) Steering Committee. Lancet. 1998;351(9111):1225-32.

2. Solé D, Yamada E, Vana AT, Costa-Carvalho BT, Naspitz CK. Prevalence of asthma and related symptoms in school-age children in São Paulo, Brazil--International Study of Asthma and Allergies in Children (ISAAC). J Asthma. 1999;36(2):205-12.

3. Brasil. Secretaria Nacional de Ações Básicas de Saúde. Estatística de Mortalidade, Brasil. Brasília: Ministério da Saúde; 2005.

4. Brasil. Secretaria Nacional de Ações Básicas de Saúde. Estatística de Mortalidade, Brasil. Brasília: Ministério da Saúde; 2004.

5. Sociedade Brasileira de Pneumologia e Tisiologia. Il Consenso Brasileiro sobre Doença Pulmonar Obstrutiva Crônica. J Bras Pneumol. 2004;30(Suppl 5):S1-S42.

6. Menezes AM, Jardim JR, Pérez-Padilla R, Camelier A, Rosa F, Nascimento 0, et al. Prevalence of chronic obstructive pulmonary disease and associated factors: the PLATINO Study in São Paulo, Brazil. Cad Saude Publica. 2005;21(5):1565-73.

7. Rabe KF, Hurd S, Anzueto A, Barnes PJ, Buist SA, Calverley P, et al. Global Strategy for the Diagnosis, Management, and Prevention of Chronic Obstructive Pulmonary Disease: GOLD executive summary. Am J Respir Crit Care Med. 2007;176(6):532-55. Epub 2007 May 16.

8. Global Initiative for Chronic Obstructive lung Disease (GOLD). Global strategy for the diagnosis, management, and prevention of chronic obstructive pulmonary disease. Bethesda (MD): Global Initiative for Chronic Obstructive Lung Disease, World Health Organization, National Heart, Lung and Blood Institute; 2007.

9. Laube BL. In vivo measurements of aerosol dose and distribution: clinical relevance. J Aerosol Med. 1996;9 Suppl 1:S77-91.

10. Muchão FP, Perín SL, Rodrigues JC, Leone C, Silva Filho LV. Evaluation of the knowledge of health professionals at a pediatric hospital regarding the use of metereddose inhalers [Article in Portuguese]. J Bras Pneumol. 2008;34(1):4-12.

11. Fernandes ALG, Cabral ALB, Faresin SM. 1 Consenso Brasileiro de Educação em Asma. J Pneumol. 1996;22(suppl 1):1-24.

12. Cabral AL, Carvalho WA, Chinen M, Barbiroto RM, Boueri FM, Martins MA. Are International Asthma Guidelines effective for low-income Brazilian children with asthma? Eur Respir J. 1998;12(1):35-40.

13. de Oliveira MA, Faresin SM, Bruno VF, de Bittencourt AR, Fernandes AL. Evaluation of an educational programme for socially deprived asthma patients. Eur Respir J. 1999;14(4):908-14.

14. Bettencourt AR, Oliveira MA, Fernandes AL, Bogossian M. Educação de pacientes com asma: atuação do enfermeiro. J Pneumol. 2002;28(4):193-200.
15. Sociedade Brasileira de Pneumologia e Tisiologia. IV Diretrizes Brasileiras para o Manejo da Asma. J Bras Pneumol. 2006;32(Suppl 7):S447-S474.

16. Wright J, Brocklebank D, Ram F. Inhaler devices for the treatment of asthma and chronic obstructive airways disease (COPD). Qual Saf Health Care. 2002;11(4):376-82.

17. Molimard M, Raherison C, Lignot S, Depont F, Abouelfath A, Moore N. Assessment of handling of inhaler devices in real life: an observational study in 3811 patients in primary care. J Aerosol Med. 2003;16(3):249-54.

18. Plaza V, Sanchis J. Medical personnel and patient skill in the use of metered dose inhalers: a multicentric study. CESEA Group. Respiration. 1998;65(3):195-8.

19. Steier J, Trammer T, Cloes RM, Petro W. Optical feedback training of inhalation with Autohaler and Turbuhaler in COPD patients. Lung. 2003;181(4):183-92.

20. Schulte M, Osseiran K, Betz R, Wencker M, Brand $\mathrm{P}$, Meyer T, et al. Handling of and preferences for available dry powder inhaler systems by patients with asthma and COPD. J Aerosol Med Pulm Drug Deliv. 2008;21(4):321-8.

21. Giraud V, Roche N. Misuse of corticosteroid metereddose inhaler is associated with decreased asthma stability. Eur Respir J. 2002;19(2):246-51.

22. Muniz JB, Padovani CR, Godoy l. Inalantes no tratamento da asma: avaliação do domínio das técnicas de uso por pacientes, alunos de medicina e médicos residentes. J Pneumol. 2003;29(2):75-81.

23. Virchow JC, Crompton GK, Dal Negro R, Pedersen S, Magnan A, Seidenberg J, et al. Importance of inhaler devices in the management of airway disease. Respir Med. 2008;102(1):10-9.

24. Stelmach R, Robles-Ribeiro PG, Ribeiro M, Oliveira JC, Scalabrini A, Cukier A. Incorrect application technique of metered dose inhalers by internal medicine residents: impact of exposure to a practical situation. J Asthma. 2007;44(9):765-8.

25. Chopra N, Oprescu N, Fask A, Oppenheimer J. Does introduction of new "easy to use" inhalational devices improve medical personnel's knowledge of their proper use? Ann Allergy Asthma Immunol. 2002;88(4):395-400.

26. Sandrini A, Jacomossi A, Farensin SM, Fernandes AL, Jardim JR. Aprendizado do uso do inalador dosimetrado após explicação por pneumologista. J Pneumol. 2001;27(1):7-10.

27. van der Palen J, Klein JJ, van Herwaarden $\mathrm{CL}$, Zielhuis GA, Seydel ER. Multiple inhalers confuse asthma patients. Eur Respir J. 1999;14(5):1034-7.

28. Lavorini F, Fontana GA. Targeting drugs to the airways: The role of spacer devices. Expert Opin Drug Deliv. 2009;6(1):91-102.

29. Escribano A, Tutuncu A, Löhr l, Carlholm M, Polanowski T. Clinical comparability between the CFC and HFA budesonide pressurised metered-dose inhalers in paediatric patients with asthma: a randomised controlled trial. Curr Med Res Opin. 2006;22(6):1085-92.

30. Costa Mdo R, Oliveira MA, Santoro IL, Juliano Y, Pinto JR, Fernandes AL. Educational camp for children with asthma. J Bras Pneumol. 2008;34(4):191-5. 


\section{Sobre os autores}

Maria Luiza de Moraes Souza

Graduanda em Medicina. Faculdade de Medicina de Ribeirão Preto, Universidade de São Paulo, Ribeirão Preto (SP) Brasil.

\section{Andrea Cristina Meneghini}

Fisioterapeuta. Hospital das Clínicas, Faculdade de Medicina de Ribeirão Preto, Universidade de São Paulo, Ribeirão Preto (SP) Brasil.

\section{Érica Ferraz}

Fisioterapeuta. Hospital das Clínicas, Faculdade de Medicina de Ribeirão Preto, Universidade de São Paulo, Ribeirão Preto (SP) Brasil.

\section{Elcio Oliveira Vianna}

Professor Associado. Departamento de Clínica Médica, Faculdade de Medicina de Ribeirão Preto, Universidade de São Paulo, Ribeirão Preto (SP) Brasil.

\section{Marcos Carvalho Borges}

Professor Adjunto. Universidade Federal de São Carlos, São Carlos (SP) Brasil. 